\title{
Investigating critical systems via the distribution of correlation lengths
}

\author{
Lorenzo Palmieri ${ }^{*}$ \\ Centre for Complexity Science and Department of Mathematics, Imperial College London, \\ South Kensington Campus, SW7 2AZ London, United Kingdom \\ Henrik Jeldtoft Jensen ${ }^{\dagger}$ \\ Centre for Complexity Science and Department of Mathematics, Imperial College London, \\ South Kensington Campus, SW7 2AZ London, United Kingdom \\ and Institute of Innovative Research, Tokyo Institute of Technology, 4259 Nagatsuta-cho, Yokohama 226-8502, Japan
}

(Received 14 October 2019; accepted 16 January 2020; published 24 February 2020)

\begin{abstract}
We introduce a novel approach to study the critical behavior of equilibrium and nonequilibrium systems, which is based on the concept of an instantaneous correlation length. We analyze in detail two classical statistical mechanical systems (the XY model and the Ising model) and one of the prototype models of self-organized criticality [the forest fire model (FFM)]. The proposed method can both capture the critical behavior of the XY model and the Ising model and discriminate between the nature of the phase transition in the two scenarios. When applied to the FFM, it gives surprising results, suggesting that the model could be critical despite displaying broken scaling in the distribution of cluster sizes.
\end{abstract}

DOI: 10.1103/PhysRevResearch.2.013199

\section{INTRODUCTION}

The concept of criticality is widely used in many disciplines, spanning finance [1-4], meteorology [5-7], neuroscience [8-11], and physics [12-15]. A system in a critical state is usually characterized by scale invariance and selfsimilarity and by the development of strong instabilities which are caused by the emergence of long-range temporal or spatial interactions. In statistical physics, the term criticality indicates the behavior of a system near a critical point, which is typically associated with a phase transition between two different states. A classic example of phase transition is the behavior of magnets near a critical temperature $T_{c}$, which separates an ordered state at low temperatures $\left(T<T_{c}\right)$ from a disordered one at high temperatures $\left(T>T_{c}\right)$. When a system is in a critical state, it is highly susceptible to external perturbations, and it is characterized by the emergence of long-range correlations between its constituent components. This high susceptibility is a direct consequence of the self-similarity of the correlation function, which emerges from microscopic interactions and leads to the presence of strong correlations on all scales of the system.

The distinctive nature of the critical state is the reason why it often attracts attention both theoretically and in real systems. Here we mention a few examples from very different systems, namely the spatial distribution of vegetation $[16,17]$,

\footnotetext{
*1.palmieri16@imperial.ac.uk

†h.jensen@imperial.ac.uk

Published by the American Physical Society under the terms of the Creative Commons Attribution 4.0 International license. Further distribution of this work must maintain attribution to the author(s) and the published article's title, journal citation, and DOI.
}

the solar wind [18], and the distribution of activity across the brain [19]. The method developed in the present paper is of relevance in this respect because of its simplicity and generality. It applies to models or real systems, in or out of equilibrium, whenever one has access to snapshots describing the evolution of the spatial configurations and allows to extrapolate the large system's behavior by analyzing configurations of different sizes.

In statistical physics, the correlation function is usually defined as the difference between the canonical ensemble average $\langle\ldots\rangle$ of the scalar product between two random variables $s_{1}$ and $s_{2}$, which might denote, say, spins at position $r_{0}$ and $r_{0}+r$ or the displacement of particles at these positions with respect to some lattice structure and their uncorrelated average product:

$$
C(r)=\left\langle s_{1}\left(r_{0}\right) s_{2}\left(r_{0}+r\right)\right\rangle_{r_{0}}-\left\langle s_{1}\left(r_{0}\right)\right\rangle_{r_{0}}\left\langle s_{2}\left(r_{0}+r\right)\right\rangle_{r_{0}} .
$$

Introducing the external control parameter $X$, in the thermodynamic limit one expects to find a power-law behavior when $X$ is tuned to a critical value $X_{c}$ :

$$
C\left(r \mid X_{c}\right) \sim r^{-\eta} .
$$

Equation (2) implies that the relative change in correlation is independent of the scale of $r$, namely

$$
\frac{C\left(\mu r \mid X_{c}\right)}{C\left(r \mid X_{c}\right)} \sim \mu^{-\eta} .
$$

The fact that correlations are present at all scales translates into the existence of long-range correlations and the resulting critical behavior of the whole system. Away from the critical point, correlations typically decay as an exponential function, and the characteristic length of the exponential is referred to as the correlation length $\bar{\xi}$. The typical functional form that is 
assumed for the correlation function near a critical point is

$$
C(r \mid X, L) \sim r^{-\eta} e^{-\frac{r}{\xi(X, L)}},
$$

where $\bar{\xi}$ indicates the typical length over which two positions in the system are correlated and depends on the control parameter of the system $X$ and the system size $L$. This length is limited by $L$ and diverges in the thermodynamic limit in correspondence of the critical value of the control parameter $X_{c}$, i.e., $\bar{\xi}\left(X_{c}, L \rightarrow \infty\right) \rightarrow \infty$, giving Eq. (2). It is clear then that the correlation length act as a parameter that describes the typical extension of correlations inside a system and therefore represents the most reasonable quantity to look at when one investigates the critical behavior of a physical system. However, it is essential to observe that measuring a diverging correlation length is not enough to determine whether a system is in a critical state because it does not convey any information about the scaling behavior (in the sense of self-similarity or invariance under change of scale) of the system. In other words, one could observe a divergent correlation length even in a system that is not scale invariant and therefore not critical, as will be discussed in the next sections. In this paper, we introduce a new method to investigate the critical behavior of a system. This method is still based on the study of the correlation function but introduces a new correlation length that is no longer a parameter of the system, but a stochastic variable whose distribution is able to catch at the same time the scale invariance of the system, the asymptotic behavior of the correlation length and the universal properties of the model.

\section{THE INSTANTANEOUS CORRELATION LENGTH FORMALISM}

The method proposed is based on the instantaneous correlation length introduced in Ref. [20]. For simplicity, we consider models defined on a two-dimensional (2D) lattice consisting of a "spin" variable $s(r)$ on each of the $L^{2}$ lattice positions $r$. We sample $N$ independent lattice configurations $S_{1}, S_{2}, \ldots, S_{N}$ during the time evolution. The classic estimate of the correlation length $\bar{\xi}$ goes as follows: For each configuration $S_{t}$ one computes the two-point correlation function $C_{t}(r)$ between two spins $s_{1}$ and $s_{2}$ at positions $r_{0}$ and $r_{0}+r$. Assuming translational invariance, one has:

$$
C_{t}(r)=\left\langle s_{1}\left(r_{0}, t\right) s_{2}\left(r_{0}+r, t\right)\right\rangle_{r_{0}}-\left\langle s\left(r_{0}, t\right)\right\rangle_{r_{0}}^{2},
$$

where the average $\langle\ldots\rangle$ is taken summing over all the possible pairs of spins and values of $r_{0}$ at a time $t$. Traditionally, one estimates the ensemble-averaged correlation function in Eq. (1) by taking the time average of Eq. (5). Iterating this procedure for different configurations, one obtains an ensemble of correlation functions $\left\{C_{1}, C_{2}, \ldots, C_{N}\right\}$, which can be used to compute the time-averaged correlation function $\bar{C}(r)$ for which the following functional form is usually assumed near a critical point:

$$
\bar{C}(r \mid X, L) \sim r^{-\eta} e^{-\frac{r}{\xi(X, L)}},
$$

where $\bar{\xi}(X, L)$ is the correlation length which depends on a control parameter $X$ and the system size $L$. In correspondence of the critical value of the control parameter $X=X_{c}$, the correlation length $\bar{\xi}\left(X_{c}, L\right)$ diverges in the thermodynamic limit, and the correlation function decays algebraically.

Now we introduce the instantaneous correlation length formalism. Assuming that the system size is sufficiently large to give reasonable statistics for the instantaneous correlation function $C_{t}(r)$, one can fit the instantaneous correlation length $\xi_{t}$ using the same functional form that is used in Eq. (6). After the fitting procedure, explained in detail in Appendix B, one obtains an ensemble of instantaneous correlation lengths $\left\{\xi_{1}, \xi_{2}, \ldots, \xi_{N}\right\}$. Each $\xi_{t}$ is a measure of how critical a single configuration $S_{t}$ is. If the system is far from a critical point, then one expects $\xi_{t}$ to be always small because the correlation function will decrease exponentially fast. On the other hand, as the system approaches $X_{c}$ there will be an increasing fraction of configurations with a big correlation length, which corresponds to the power-law prefactor in Eq. (6) dominating the behavior. Although one expects the ensemble-averaged correlation length $\bar{\xi}(X)$ and the average instantaneous correlation length $\xi$ to scale in the same way, it is essential to stress the fact that they are two distinct mathematical objects: the first being a parameter of the ensemble-averaged correlation function and the second being a stochastic variable. Indeed, the strength of this new approach lies in the fact that we can now use the ensemble of $\xi_{t}$ to compute not only the average correlation length $\langle\xi\rangle$ but also the distribution of correlation lengths $P(\xi) . P(\xi)$ is an entirely new physical object and, as we will see, contains essential information about the critical behavior of the system under analysis.

\section{THE DISTRIBUTION OF THE INSTANTANEOUS CORRELATION LENGTHS}

Using $P(\xi)$, it is possible to determine whether a system is at a critical point. This can be done by looking at the conditional probability $P(\xi \mid X)$, which should become scale invariant as $X_{c}$ is approached. Assuming simple scaling, one expects:

$$
P\left(\xi \mid X_{c}\right)=b G\left(\frac{\xi}{\xi_{c}}\right) \xi^{-\tau}
$$

for $\xi$ and $\xi_{c}$ bigger than a constant lower cutoff $\xi_{0}$. In Eq. (7), $\xi_{c}$ represents an upper cutoff that diverges in the thermodynamic limit, $G\left(\frac{\xi}{\xi_{c}}\right)$ is a universal scaling function, $\tau$ is a critical scaling exponent and $b$ is a nonuniversal metric factor. In general, the upper cutoff scales as $\xi_{c} \sim a L^{\beta}$, where $a$ is another nonuniversal metric factor and $\beta$ is related to the universal spatial dimension of the observable [21,22]. From Eq. (7) one can write the $n$th moment as

$$
\left\langle\xi^{n}\right\rangle=b \xi_{c}^{n-\tau+1} \int_{\frac{\xi_{0}}{\xi_{c}}}^{\infty} G(u) u^{n-\tau} d u .
$$

Imposing normalization ( $n=0$ ) one gets $\tau \geqslant 1$. Setting $n=1$ in Eq. (8) and absorbing the constant prefactor in $G(u)$, we can define the average correlation length as

$$
\langle\xi\rangle=L^{\beta(2-\tau)} \int_{\frac{\xi_{0}}{\xi_{c}}}^{\infty} G(u) u^{1-\tau} d u .
$$

Which is equivalent to setting $b=a^{\tau-2}$ in Eqs. (7) and (8). In the following sections, we will study the behavior of $P(\xi)$ in 
two traditional statistical mechanical systems, the Ising model and the XY model, and in one of the prototype models of selforganized criticality, the forest fire model. We conclude this section observing that if Eq. (7) holds, then it automatically allows the introduction of the new critical exponent $\tau$.

\section{ISING MODEL}

The Ising model is a mathematical model of ferromagnetism that was invented by Wilhelm Lenz in 1920 and solved for the first time in one dimension by Ernst Ising in 1925 [23,24]. The model consists of $N$ interacting two-state spin variables $\sigma_{i} \in[-1,1]$ which represent adjacent magnetic dipoles. The energy that is associated with a given configuration $\sigma$ is given by

$$
H(\sigma)=-\sum_{\langle i, j\rangle} J_{i j} \sigma_{i} \sigma_{j}-\sum_{i=1}^{N} h_{i} \sigma_{i},
$$

where the first sum is over all pairs of adjacent spins $\langle i, j\rangle, J_{i j}$ is the interaction strength and $h_{i}$ is the external magnetic field. The two-dimensional square lattice Ising model was solved in 1944 by Onsager [25] in the case of no external field $\left(h_{i}=\right.$ 0 ) and assuming periodic boundary conditions and constant interaction strength along the $x$ axis $\left(J_{x}\right)$ and the $y$ axis $\left(J_{y}\right)$. The 2D Ising model is central in statistical physics because it is one of the simplest statistical models to exhibit a phase transition between an ordered phase (low temperatures) and a disordered phase (high temperatures). In the case of isotropic interactions $J_{x}=J_{y}$, the critical value of the temperature $T=$ $T_{c}$ that marks the phase transition is given by

$$
\frac{k_{B} T_{c}}{J}=\frac{2}{\ln (1+\sqrt{2})} \simeq 2.269 .
$$

When one looks at the lattice at different temperatures, it can be noted that the high-temperature phase is characterized by disorder, because the entropy introduced in the system by the temperature destroys long-range correlations, which results in random configurations with roughly half of the spins up and half of the spins down and no emergent complex structures. On the other hand, at low temperatures, most of the spins will be able to align in order to minimize the energy, giving rise to ordered configurations. At the critical point, the correlation function decays as a power law, and we are in the presence of long-range interactions and the creation of fractal structures, as can be seen in Fig. 1. This behavior is reflected by the fact that the correlation length becomes proportional to the system size $L$ and diverges in the limit $L \rightarrow \infty$.

In terms of the instantaneous correlation length formalism, it is reasonable to assume that the upper cutoff scales like $L$, i.e., $\xi_{c} \sim L$ and hence $\beta=1$, and then in order to maintain the linear relationship between the system size $L$ and $\langle\xi\rangle$, we should have $\tau=1$ in Eq. (9), meaning that the constant of proportionality would be given by the integral of $G(u)$. Under these assumptions and in the large $L$ limit, Eq. (9) reduces to

$$
\langle\xi\rangle=L \int_{0}^{\infty} G(u) d u .
$$

As $\tau=1$ implies that the $\operatorname{limit} \lim _{u \rightarrow 0} G(u)=0$ [22]. In order to verify whether our ansatz is correct, we need to evaluate

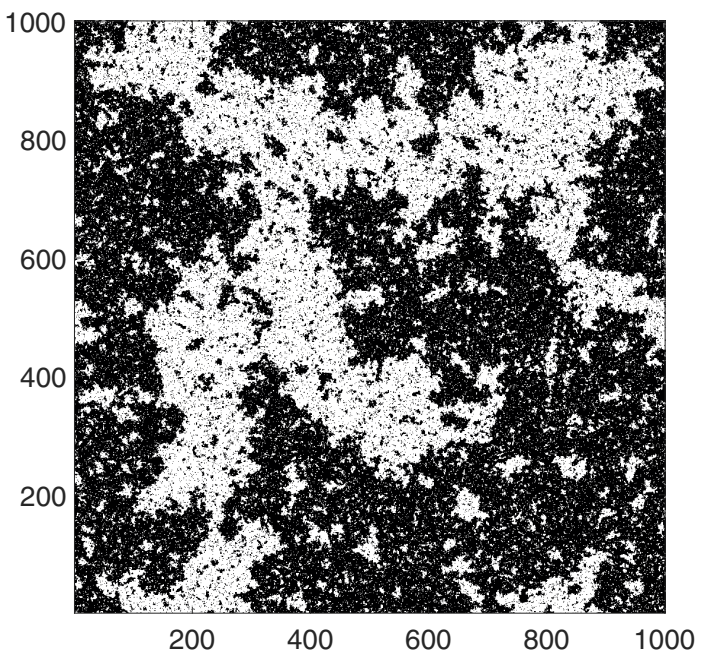

FIG. 1. Sample configuration of the 2D Ising model for a square lattice with $L=1000$ at $T_{c}$.

whether there is a value of the control parameter $X=X_{c}$ such that $P\left(\xi \mid X_{c}\right)$ becomes scale invariant. For the 2D Ising model, this corresponds to the critical temperature given by Onsager's solution in Eq. (11). In our simulations, we used the Wolff Algorithm [26] in order to reduce the critical slowing down and for each system size $L$ we sampled $10^{6}$ independent configurations to estimate $P(\xi)$ at $T_{c}$. In the following, we will use $\xi_{0}=1$ and $\xi_{c}=\frac{L}{\sqrt{2}}$ as the reference upper cutoff, as this is the maximum physical distance between two points on a square lattice with periodic boundary conditions. Plotting $P(\xi) \xi$ as a function of $\frac{\xi}{\xi}$, we can indeed perform a data

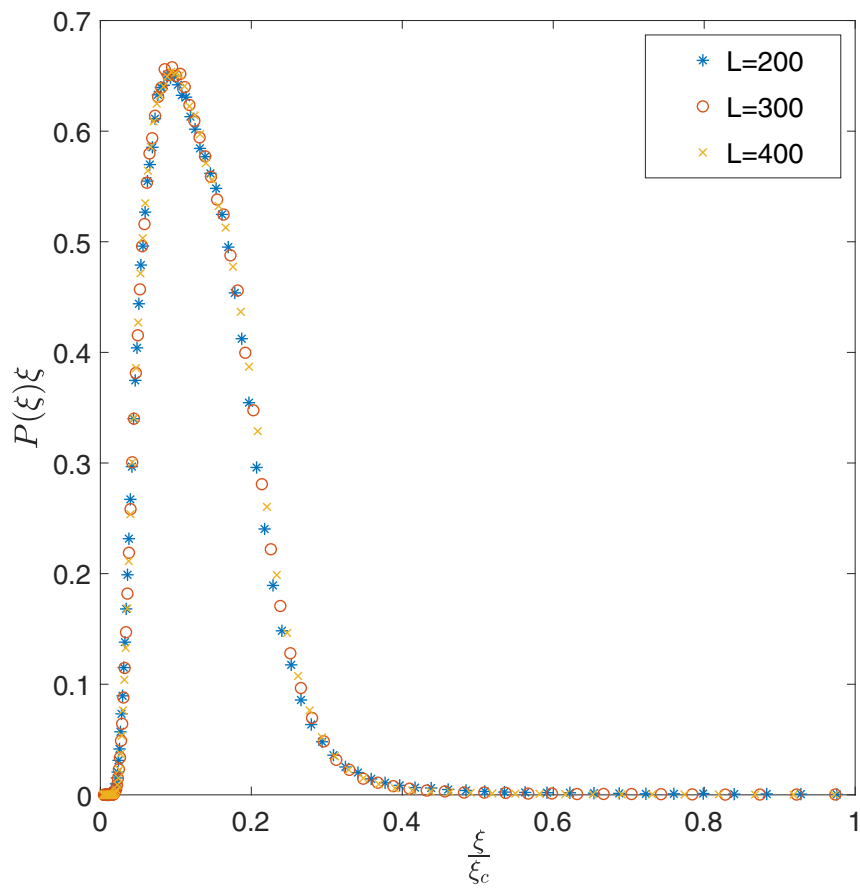

FIG. 2. Data collapse for the 2D Ising model. The distribution of correlation lengths $P(\xi)$ becomes scale invariant in correspondence of $T_{c}$ and for $\tau=1$ and $\beta=1$. 

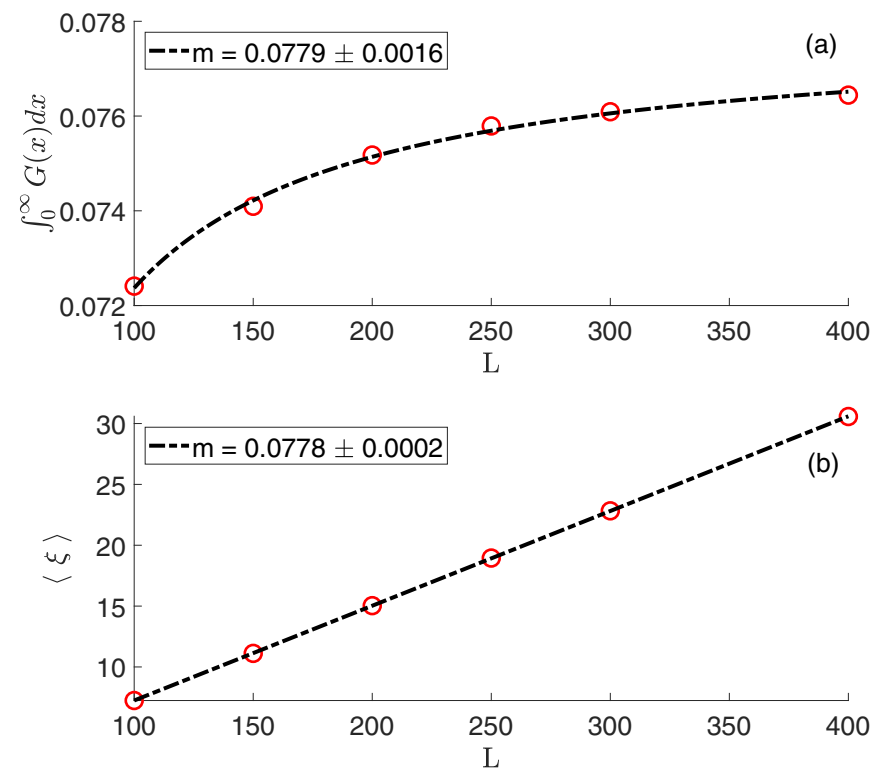

FIG. 3. Estimate of the constant of proportionality $m$ between $\langle\xi\rangle$ and $L$ for the 2D Ising model. In panel (a), $m$ is estimated by fitting the integral of $G(x)$ vs $L$ with a power law. In panel (b), $m$ is estimated by means of a linear fit of $\langle\xi\rangle$ vs. $L$. The integral in (a) converges toward the asymptotic value as $L^{-\lambda}$, with $\lambda=1 \pm 0.5$. The error corresponds to confidence bounds of $95 \%$.

collapse at $T_{c}$ for $\tau=1$ and $\beta=1$ (Fig. 2). The resulting curve corresponds to $\sqrt{2} G(u)$ and its integral can be used to compute the proportionality constant between $\langle\xi\rangle$ and $L$ in Eq. (12). Figure 3 shows how the integral of $G(x)$ converges to a value that is consistent with the estimated gradient of the line $\langle\xi\rangle=m L$.

In summary, when applied to the 2D Ising model, our method was able to identify the critical temperature as the $T$ for which $P(\xi \mid T)$ becomes scale invariant and to capture the scaling behavior of $\langle\xi\rangle$, which is consistent with the classical theory. In addition to these two well-known results, we were able to introduce a new critical exponent for the Ising model, i.e., $\tau=1$, and to relate the rate of growth of the correlation length to the integral of $G(u)$.

\section{XY MODEL}

The two-dimensional XY model is a paricular case of the Heisenberg model, which was introduced in 1928 [27] as a model for ferromagnetism. Similarly to the Ising model, it consists of a system of spins on a lattice, with the difference that the individual spins can rotate in any direction in the two-dimensional XY spin plane, i.e., $\vec{\sigma}_{i}=\left(\sigma_{x}, \sigma_{y}\right)$, and are not constrained to take only two values. The energy of the model is given by

$$
H(\sigma)=-\sum_{\langle i, j\rangle} J_{i j} \vec{\sigma}_{i} \cdot \vec{\sigma}_{j}=-\sum_{\langle i, j\rangle} J_{i j} \cos \left(\theta_{i}-\theta_{j}\right),
$$

where the first sum is over pairs of adjacent spins $\langle i, j\rangle, J_{i j}$ is the interaction strength, and $\theta_{i}$ is the angle that a spin $\vec{\sigma}_{i}$ makes with respect to some arbitrary direction in the lattice plane. As for the Ising model, in our simulation we keep

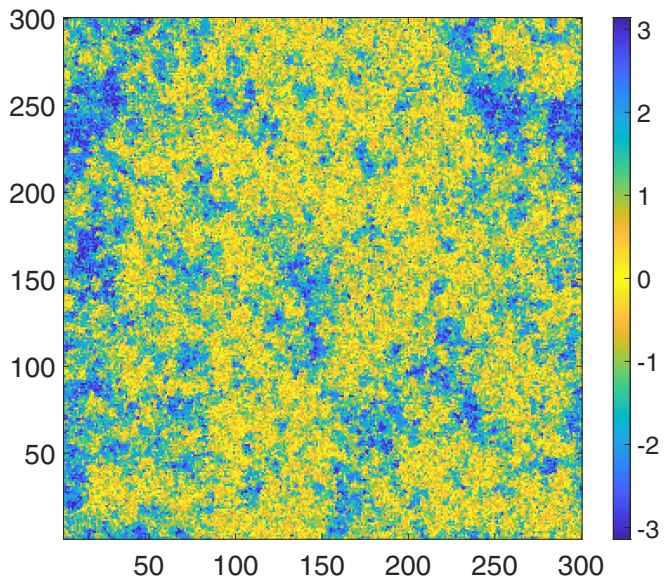

FIG. 4. Sample configuration of the 2D XY model with $L=300$ at $T_{\mathrm{KT}}$. The range of angles $[-\pi, \pi]$ has been mapped to a periodic spectrum of colors for better representation.

the interaction strengths constant $J_{i j}=J$ and apply periodic boundary conditions. A typical realization of the model is represented in Fig. 4.

The two-dimensional version of this model is well known for the peculiar behavior of the correlation function, which decays exponentially fast at high temperatures, and as a power law at low temperatures [28], even though the overall magnetization remains zero. This peculiar transition is named after Kosterlitz and Thouless who first discovered it in 1973 [29]. The XY model is a relevant case to discuss in our context because of the behavior of the correlation length, which diverges even for finite systems at temperatures below the Kosterlitz-Thouless temperature $T_{\mathrm{KT}} \simeq 0.892 J$ [30-32]. In the XY model, the two-point correlation function is defined as [33]

$$
C(r)=\left\langle\cos \left[\theta\left(r_{0}\right)-\theta\left(r_{0}+r\right)\right]\right\rangle .
$$

In our simulations we used the Wolff algorithm [26] sampling $10^{5}$ independent configurations to estimate $P(\xi)$ at $T_{\mathrm{KT}}$ and used $\xi_{0}=1$ and $\xi_{c}=\frac{L}{\sqrt{2}}$. As for the Ising model, it is possible to perform a data collapse for $P(\xi)$ in correspondence of $T_{\mathrm{KT}}$ and for $\tau=1$ and $\beta=1$. Although the Ising model and the XY model share the same exponents, we can observe in Figs. 5 and 6 that in the XY model, $\xi$ is able to exceed the system size $L$. This is in line with the theory, which predicts a pure power law in two dimensions in correspondence of $T_{\mathrm{KT}}$ [28]. The presence of the Kosterlitz-Thouless phase transition and the behavior of the correlations is summarized in Fig. 7, where we plot the probability $P(\xi>L)$ at different temperatures. As one lowers the temperature, the fraction of correlation lengths that exceed the system size goes from 0 to $90 \%$, which corresponds to the pure power-law decay of correlations at $T<T_{\mathrm{KT}}$.

\section{FOREST FIRE MODEL}

The last model we consider is an out-of-equilibrium one, and it is one of the prototype models of self-organized criticality: the Drossel-Schwabl forest fire model (FFM) [34]. This model is different from the Ising model and the XY 


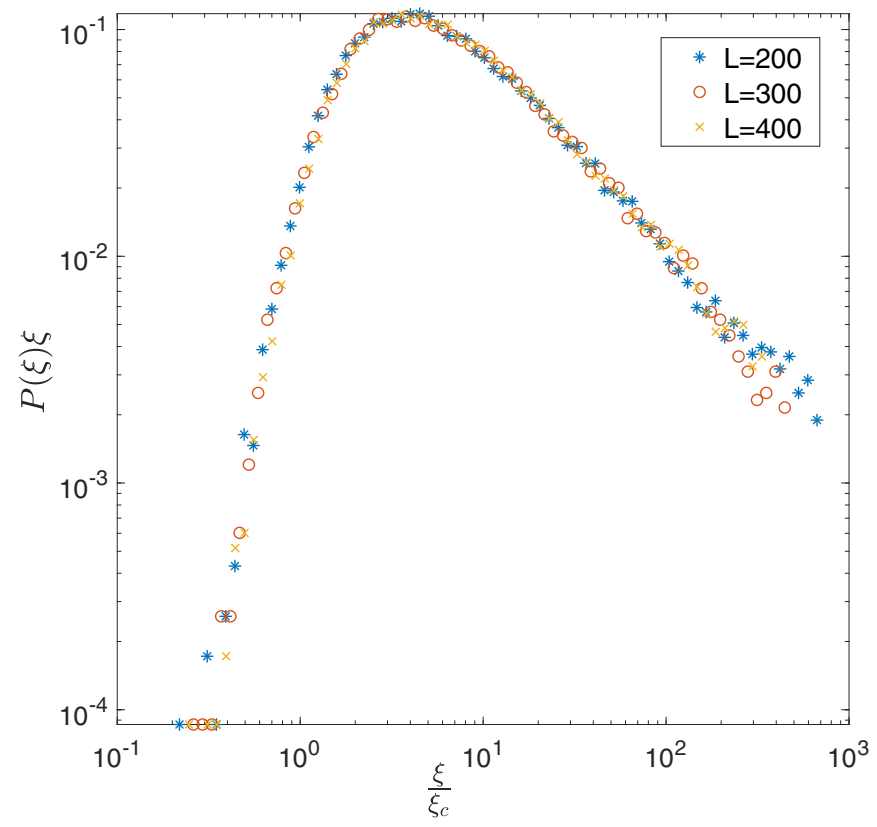

FIG. 5. Data collapse for the 2D XY model. The distribution of correlation lengths $P(\xi)$ becomes scale invariant in correspondence of $T_{\mathrm{KT}}$ and for $\tau=1$ and $\beta=1$.

model because it entails a dissipative dynamics and does not have an external control parameter, like temperature, that can be fine-tuned in order to reach a critical state. The dynamic involves the occupation of empty sites on a 2D grid with new trees (planting steps) and the removal of entire clusters of trees (burning steps). The creation of new trees and the removal of clusters results in the typical patchy appearance of the lattice, which is characterized by the presence of patches of different
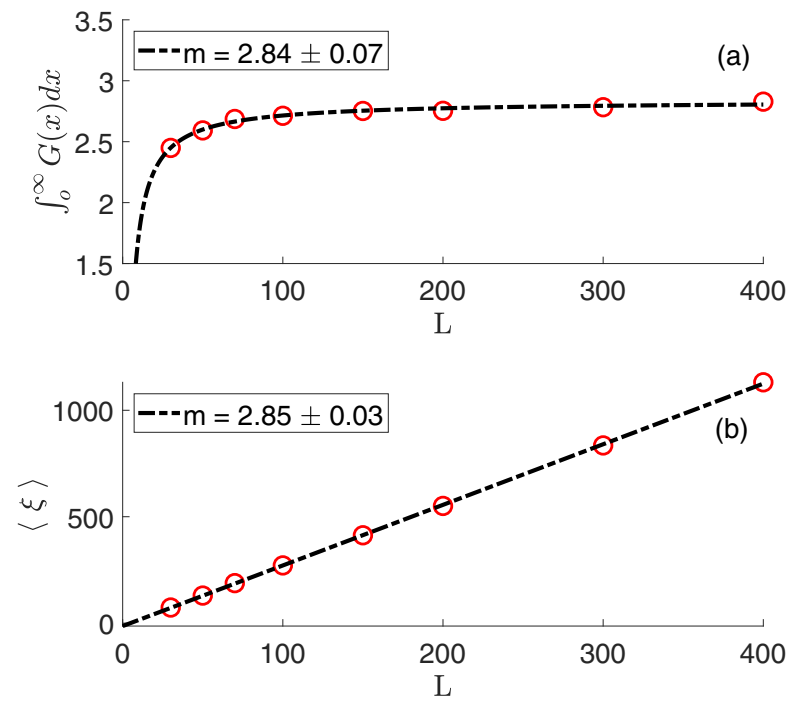

FIG. 6. Estimate of the constant of proportionality $m$ between $\langle\xi\rangle$ and $L$ for the 2D XY model. In panel (a), $m$ is estimated by fitting the integral of $G(x)$ vs $L$ with a power law. In panel (b), $m$ is estimated by means of a linear fit of $\langle\xi\rangle$ vs $L$. The integral in (a) becomes constant asymptotically as $L^{-\lambda}$, with $\lambda=1 \pm 0.5$. The error corresponds to confidence bounds of $95 \%$.

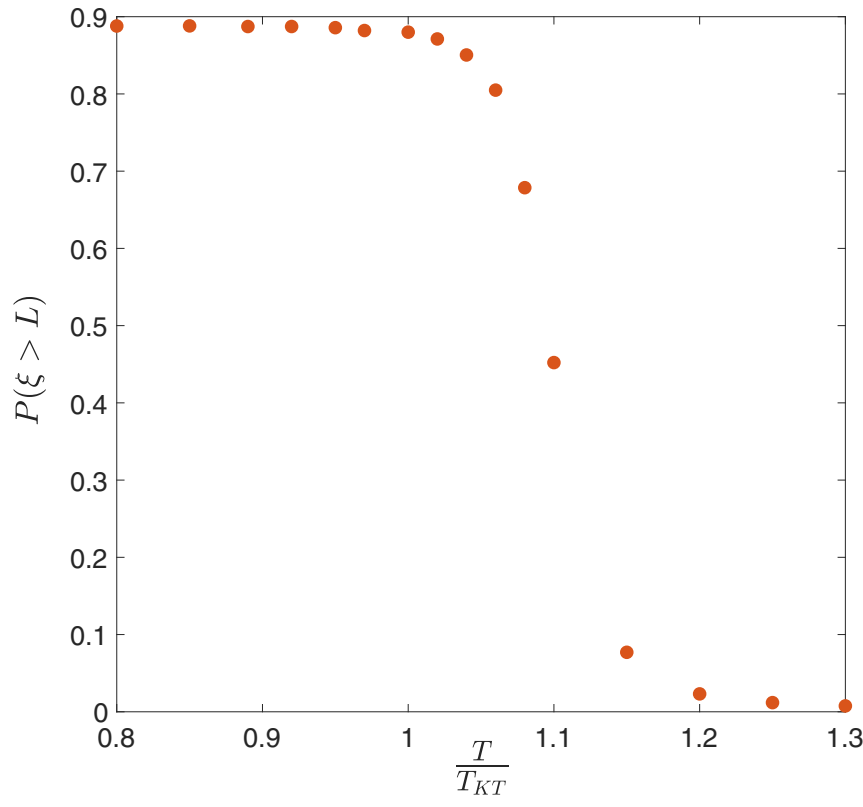

FIG. 7. Kosterlitz-Thouless phase transition in the 2D XY model with $L=100$. The fraction of correlation lengths that exceeds the system size goes from 0 to 1 as the temperature approaches $T_{\mathrm{KT}}$.

densities (Fig. 8). As in the XY model and the Ising model, we use periodic boundary conditions.

The way we implement the FFM follows [35-38] and is concisely summarized by the following pseudocode:

\section{Algorithm 1 Forest Fire Model}

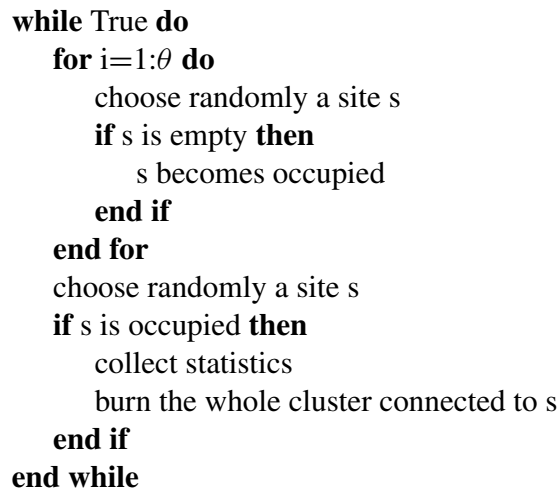

To estimate $P(\xi)$, we collected $10^{6}$ independent configurations after a transient of $5 \times 10^{6}$ burning steps. From Algorithm 1 it is clear that two parameters must be considered: the number of trees that one tries to plant $\theta$ and the system size $L$. In order to reach a critical state one would like to have both $L$ and $\theta$ infinitely large, although there is not a clear rule about how to tune $\theta$ for a finite system, and in the literature different authors have used quite a large span of $\theta$ values for the same system size $L[38,39]$. Despite the model being introduced as critical, it was subsequently realized that the observed power law in the distribution of clusters sizes displayed deviations from perfect scaling for large system sizes [38,39], implying that the model is not critical in the 


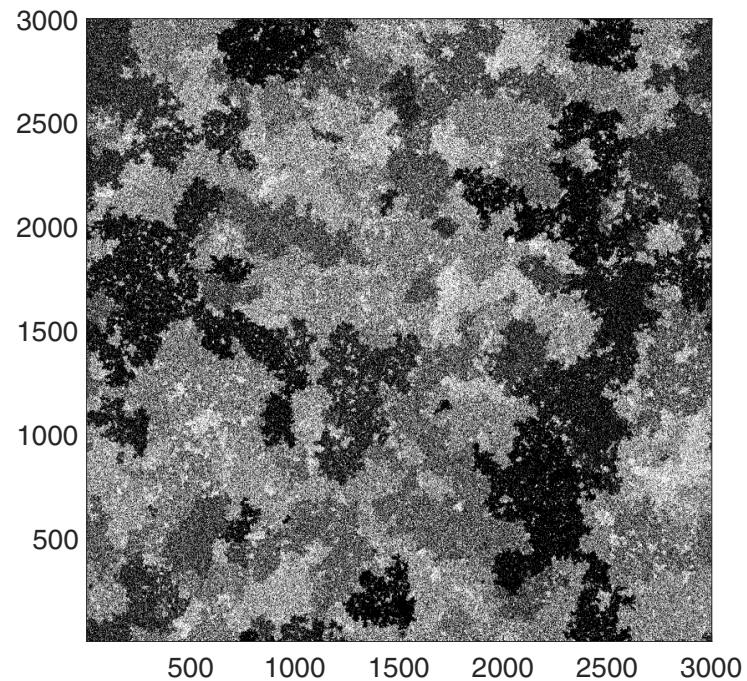

FIG. 8. Sample configuration of the 2D forest fire model for $L=3000$.

sense of being scale free [38] and that all proposed scaling laws seem to be just transient [35]. The correlation length was first studied in Ref. [40] for systems sizes $L$ and $\theta$ up to $L=512$ and $\theta=2048$, finding that $\xi \sim \theta^{v}$, with $v=0.56$. The authors also studied the connected correlation function finding $v_{c}=0.58$ and attributed this discrepancy between the two exponents to numerical error. Another estimate for larger system sizes was given in Ref. [41], where the authors used up to $L=17408$ and $\theta=10^{4}$ finding $v=0.541$ and $v_{c}=0.576$ to be statistically inconsistent and therefore concluding that the model presents two different diverging correlation lengths. This finding points in the same direction as the lack of scaling observed in the distribution of cluster sizes. However, as noted in Ref. [35], there seem to be small deviations from a power law in Fig. 1 of Ref. [41], meaning that the estimate of $v$ would be unreliable and therefore not suitable to confirm the presence of multiple diverging correlation lengths. Now we want to apply the instantaneous correlation length formalism to investigate whether $P(\xi)$ displays broken scaling as one should expect from a noncritical model. A similar approach was adopted in Ref. [20], where the critical exponent was obtained by fitting the tail of $P(\xi)$. However, the tail includes contributions from the universal function $G(\xi, L)$ and therefore that estimate of the critical exponent is spurious.

\section{A. Critical behavior in the forest fire model}

As we discussed in the previous section, it is not clear how to tune the system size $L$ and $\theta$. In previous studies on the correlation length, the standard procedure consisted in keeping the system size $L$ fixed and looking at the behavior of the correlation length as a function of $\theta$ [40,41]. Following this approach, it turns out that it is impossible to perform a data collapse for $P(\xi)$, which agrees with the general lack of scaling observed in the literature so far. The same broken scaling can be observed keeping $\theta$ fixed and changing the value of $L$. If we consider the correlation length as a surface in the space of parameters $\xi(\theta, L)$, then to keep one of the two dimensions fixed corresponds to two different ways of

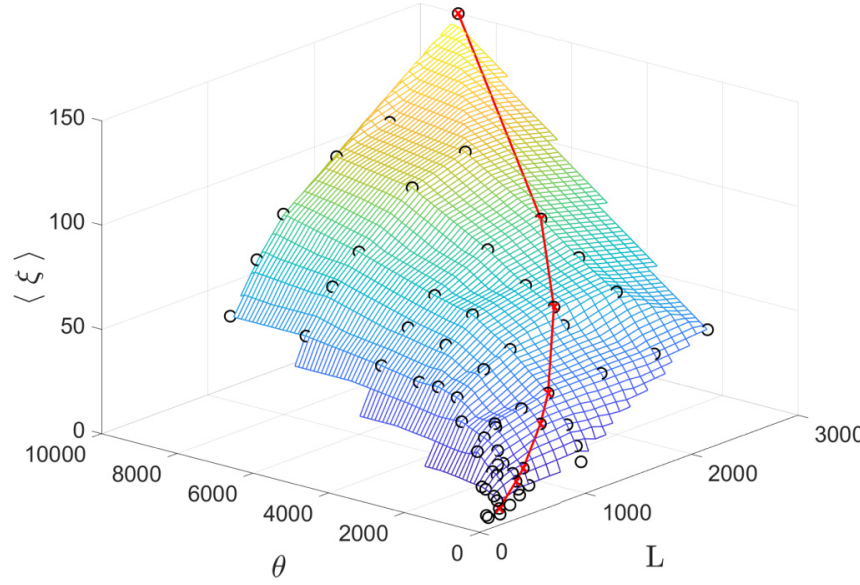

FIG. 9. $\langle\xi\rangle$ as a function of $\theta$ and $L$. The black circles correspond to the numerical simulations that have been performed to extrapolate the surface, while the red line on the surface corresponds to the path $\frac{\theta}{L^{2}}=10^{-3}$.

crossing this surface. In particular, increasing the system size $L$ without a suitable rescaling of the parameter $\theta$ could lead to a different statistical behavior of the system, although most observables like the average density of trees or the average cluster size seem to be quite robust for a wide range of $\theta$ at a fixed $L$. Even though there are infinitely many ways of coupling $\theta$ and $L$, it is sensible to choose $\frac{\theta}{L^{2}}=k$ for a constant $k$ ( $k=10^{-3}$ in our simulations). In this way, for different system sizes, one tries to plant the same fraction of trees, which seems to be reasonable if one wants to assure statistical consistency at different values of $L$. This particular path choice is shown in Fig. 9. Surprisingly, coupling the value of $\theta$ and $L$ in this way allows for a data collapse of $P(\xi)$, making $P(\xi)$ the first scale-invariant distribution observed in the forest fire model so far. Using $\xi_{0}=1$ and $\xi_{c}=\frac{L^{\beta}}{\sqrt{2}}$, we can perform a data collapse for $\tau=1$ and $\beta=1.12$ (Fig. 10). We also

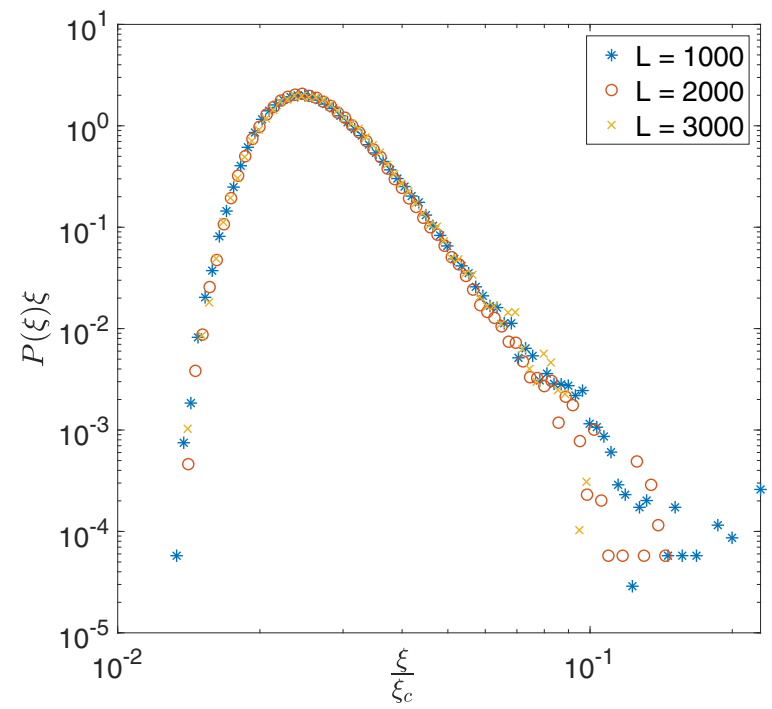

FIG. 10. Data collapse for the 2D forest fire model with $\frac{\theta}{L^{2}}=$ $10^{-3}$. The distribution $P(\xi)$ becomes scale invariant in correspondence of $\tau=1$ and $\beta=1.12$. 

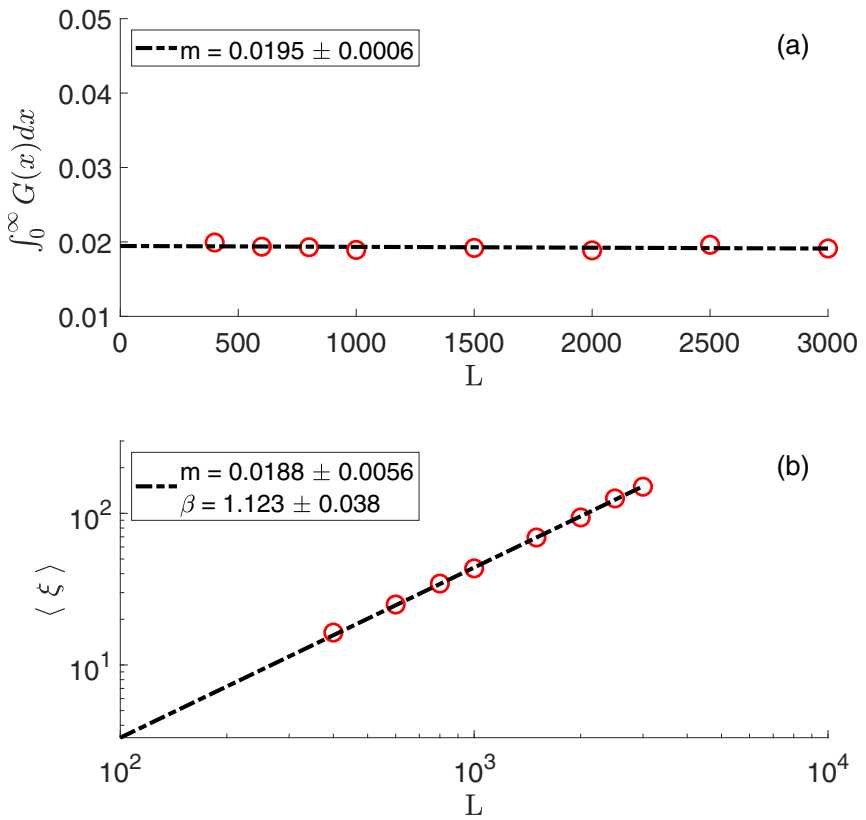

FIG. 11. Estimate of the constant of proportionality $m$ between $\langle\xi\rangle$ and $L^{\beta}$ for the 2D forest fire model. In panel (a), $m$ is estimated by fitting the integral of $G(x)$ vs $L$ with a line. In panel (b), $m$ is estimated by means of a power law fit of $\langle\xi\rangle$ vs $L$. In the FFM, $\langle\xi\rangle$ grows algebraically, which results in a straight line with gradient $\beta$ in the log-log plot. The error corresponds to confidence bounds of $95 \%$.

estimated the value of $\beta$ via fit obtaining $\beta=1.123 \pm 0.038$ with a $95 \%$ confidence bound (Fig. 11). From the relationship $\frac{\theta}{L^{2}}=k$, it follows that $\beta=2 v$ and $v=0.561 \pm 0.019$. This measurement is consistent with the exponents computed for the two-point and the connected correlation lengths in previous studies [40,41]. We conclude this section observing how the broken scaling in the distribution of cluster sizes $P(S)$ found in Refs. [38,39] is not affected by the choice of keeping fixed the ratio $\frac{\theta}{L^{2}}$. This means that the distribution of cluster sizes is not scale invariant, although the distribution of correlation lengths is scale free. Therefore, even though the clusters grow in a noncritical and non-scale-free way, there seems to be some global order in terms of the correlations, which is highlighted by the scale invariance of $P(\xi)$. This is a highly nontrivial result and an aspect that surely requires further investigations.

Finally, we observe that the value $\beta=1.12$ corresponds to a faster growth with the system size $L$ of the range, $\xi$, of the correlations, than observed in the Ising and in the XY model. This superlinear growth might be due to the burning mechanism, which introduces long-range correlations in the system as a consequence of the simultaneous removal of sites that belong to the burning cluster.

\section{CONCLUSIONS}

The instantaneous correlation length formalism introduced here is able to reproduce the well-known results about the critical behavior of the Ising model and the XY model, proving that $P(\xi)$ can be used to identify the presence of a phase transition and to estimate the asymptotic behavior of the correlation length. Furthermore, the introduction of $P(\xi)$ allowed us to define a new critical exponent $\tau$, which happens to be equal to 1 for all the three models discussed in the paper. When applied to the forest fire model, this method allowed to identify a coupling of the two parameters $L$ and $\theta$ for which $P(\xi)$ is scale invariant. The scale invariance of $P(\xi)$ was unexpected as it is the first scale-free distribution observed in the model so far, and this opens once again the debate about the criticality of the forest fire model. We observe that even though the FFM is an out-of-equilibrium model, it shares the same critical exponent $\tau=1$ of the Ising model and the $\mathrm{XY}$ model, while it differs from them in terms of the critical exponent $\beta$.

From a theoretical perspective, all systems that present a critical exponent $\tau=1$ share a very elegant property, namely that the constant of proportionality between the average correlation length and the system size dependence is given by the integral of $G(u)$. In the case of the Ising model and the $\mathrm{XY}$ model, we found $\tau=1$ and $\beta=1$. This means that all the details of the two models are contained in the integral of $G(u)$, which is characteristic of the model under analysis and becomes the only relevant quantity to distinguish between the critical behavior of correlations for the Ising model and the XY model. In Appendix A, we discuss in more detail the relationship between the ensemble correlation length and the instantaneous correlation length and how it is possible to obtain the classic critical exponent for the correlation length starting from the instantaneous correlation length formalism.

Finally, we note how the presented method could be easily applied to the study of real-world phenomena, such as brain activity or rain precipitation, as the estimate of $P(\xi)$ only requires to collect different images of the system during its time evolution. The study of $P(\xi)$ in real systems could be a useful tool to assess the scale invariance of the systems under examination and to contribute to a more accurate characterization of their critical behavior.

\section{ACKNOWLEDGMENTS}

L.P. gratefully acknowledges an EPSRC-Roth scholarship (Award Reference No. 1832407) from the Department of Mathematics at Imperial College London, the HighPerformance Computing facilities provided by the Research Computing Service, and Gunnar Pruessner for very helpful conversations. Both authors discussed the results of the numerical simulations and contributed to the final version of the manuscript. L.P. performed the numerical simulations and wrote the paper.

\section{APPENDIX A: CRITICAL EXPONENT OF THE CORRELATION FUNCTION}

As is well known from classical statistical mechanics, the correlation function of the $2 \mathrm{D}$ Ising model is characterized by a critical exponent $\bar{\eta}=0.25$ [28]. It is therefore natural to investigate whether it is possible to recover this critical exponent employing the formalism we have introduced. It is worth to stress the fact that although we assume the same functional form for the instantaneous correlation function and 


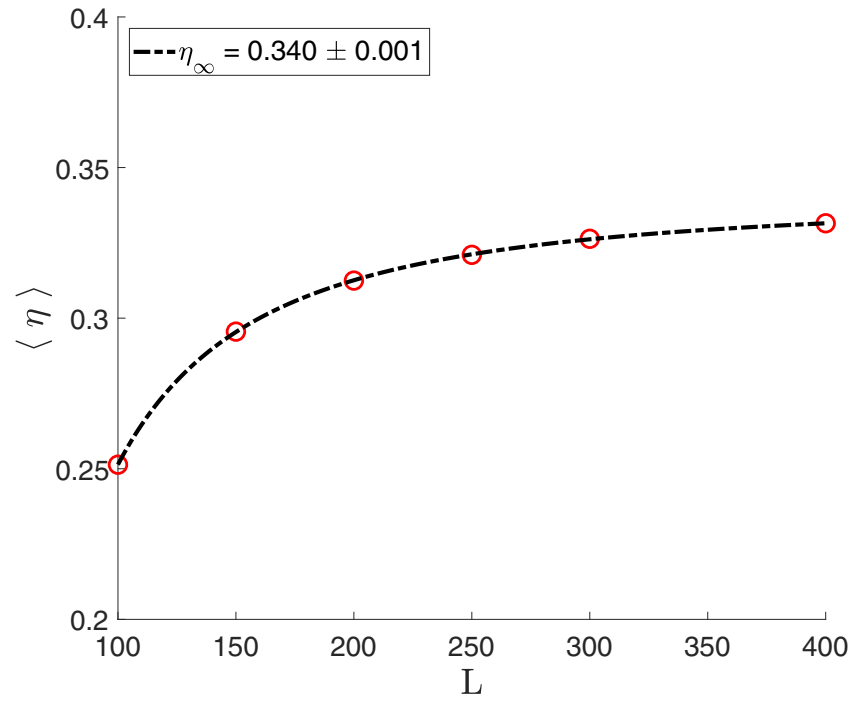

FIG. 12. The estimate of $\langle\eta\rangle$ for the 2D Ising model converges toward an asymptotic value of $\langle\eta\rangle=0.34$ as $L^{-\lambda}$ with $\lambda=1.69 \pm$ 0.05 . The error corresponds to confidence bounds of $95 \%$.

the classic one, the instantaneous values of $\xi$ and $\eta$ represent two different mathematical quantities with respect to their traditional counterpart. The crucial point is that we expect the standard correlation length $\bar{\xi}$ and $\xi$ to scale in the same way, even though the two quantities are defined differently. In particular, $\xi$ is a variable that is related to how correlated a single configuration is, and it is not bounded by the system size $L$. Regarding $\eta$, since it is an exponent, we do not expect its distribution to scale with system size but instead expect that the average of this distribution $\langle\eta\rangle$ will converge to a value

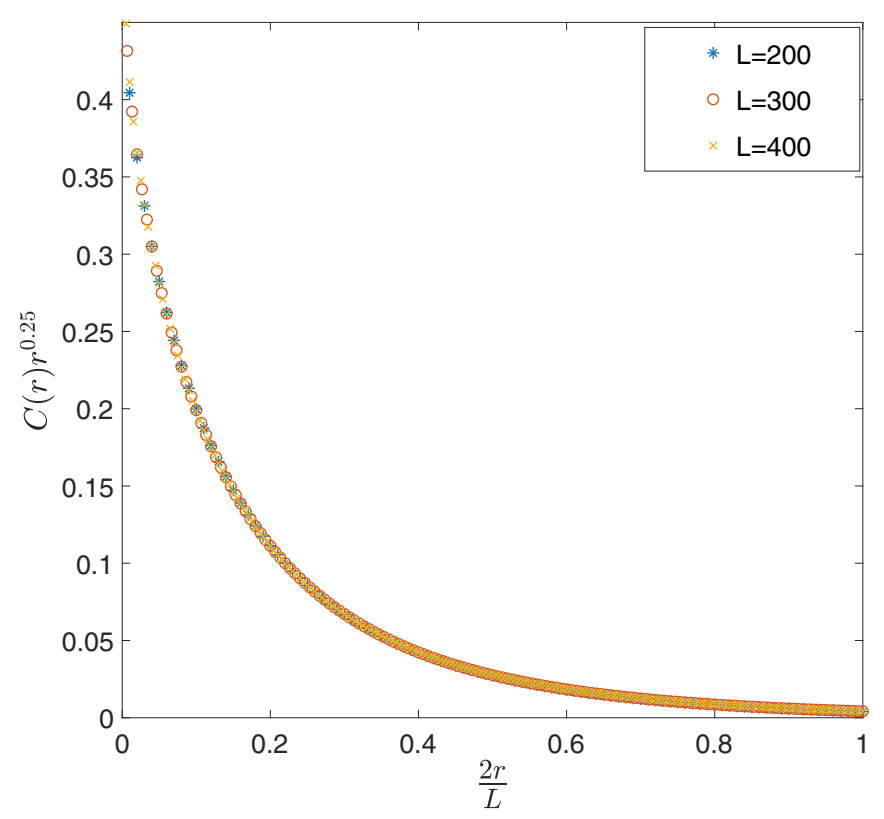

FIG. 13. It is possible to recover $\eta=0.25$ for the 2D Ising model by reverse engineering the individual correlation functions from the fitted parameters and then performing a data collapse for different values of $L$.

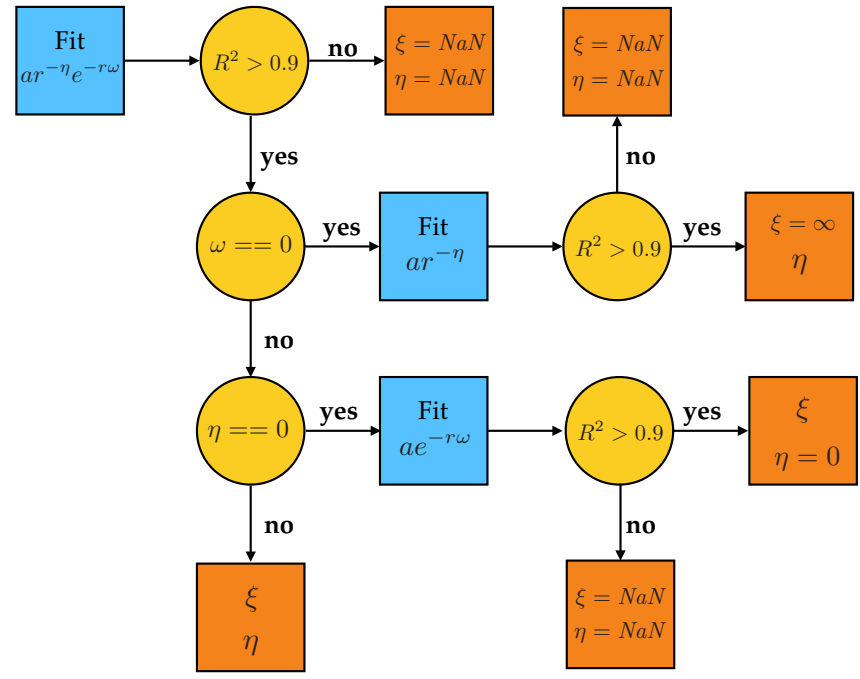

FIG. 14. Schematic representation of the fitting procedure.

that in principle is different from $\eta=0.25$ because the two quantities are averaged differently. This is confirmed by our simulations, which show that the distribution of $\eta$ is not scale invariant and that the mean value of $\eta$ tends to $\langle\eta\rangle=0.34$ as $L$ increases (Fig. 12). However, it is still possible to estimate the ensemble critical exponent $\bar{\eta}=0.25$ and, at the same time, check the accuracy of our method. In order to do so, one can use the parameters estimated via fit for each configuration $i$ and reconstruct the corresponding correlation function $C_{i}(r)$. If the error that we do in fitting $C_{i}(r)$ is negligible, then we should be able to compute the classical correlation function averaging over all configurations and hence recover $\bar{\eta}=0.25$. Indeed, plotting $C(r) r^{0.25}$ vs. $\frac{2 r}{L}$ for different system sizes we can perform a data collapse (Fig. 13), meaning that the fitting error is negligible and that we can safely recover the ensemble critical exponent $\bar{\eta}=0.25$.
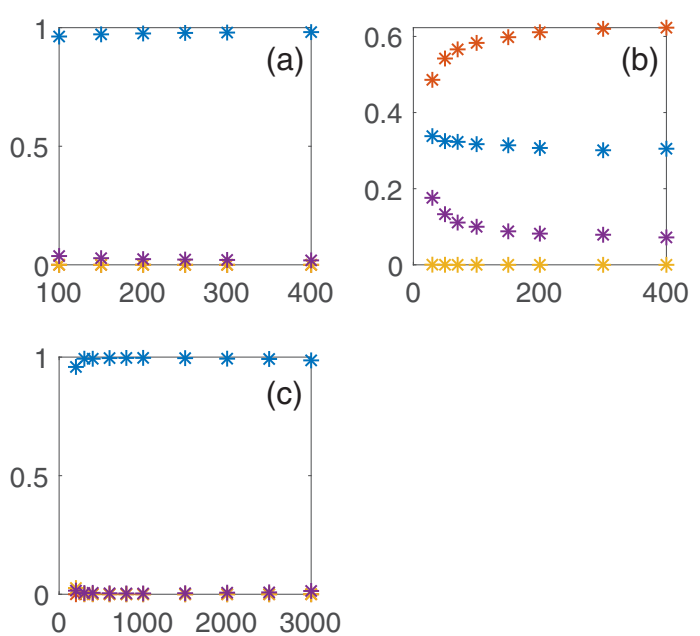

FIG. 15. Fraction of configurations that follow Eq. (4) (blue), are pure power laws (red), have $R^{2}<0.9$ (purple), or are pure exponentials (yellow). On the $x$ axis is represented the system size $L$. The three figures correspond to the Ising model (a), the XY model (b), and the forest fire model (c). 


\section{APPENDIX B: THE FITTING PROCEDURE}

The fitting procedure that we have implemented follows the logic presented in Fig. 14, where we use the inverse of the correlation length $\omega=\frac{1}{\xi}$ as a fitting parameter. The fitting algorithm goes as follows. First, we try to fit the correlation function with Eq. (4), checking that the coefficient of determination $R^{2}$ is bigger than 0.9. After the fit, if $\omega$ is fixed at zero (i.e., the quality of the fit is constantly improving by making $\omega$ closer to zero), then we perform a new fit using a pure power law and set $\xi=\infty$. If $\omega$ is well behaved and $\eta$ is fixed at zero, then we make a new fit with a pure exponential, setting $\eta=0$. If at any point of the fitting procedure $R^{2}<0.9$, then we reject the fit.

In most cases, this procedure might be regarded as overcautious. However, there are situations, like in the $X Y$ model, where one might have a pure power law even for finite systems. Although we cannot use pure power-law configurations to build the histogram of $P(\xi)$, it is convenient to keep track of these configurations to get a better understanding of the behavior of the system. In Fig. 15, we represent for each model the fraction of configurations that follow Eq. (4) (blue), are pure power laws (red), and have $R^{2}<0.9$ and thus are rejected (purple) or are pure exponentials (yellow). It turns out that for the Ising model (15a) and the forest fire model (15c) the rejection rate is low, and the vast majority of the configurations follow Eq. (4). On the other hand, the XY model shows richer behavior. We found that around $60 \%$ of the configurations are better fitted by a pure power law, while roughly $30 \%$ of the configurations follow Eq. (4) and thus are used to analyze $P(\xi)$. Finally, we observe that for the $\mathrm{XY}$ model, the rejection rate is higher than in the Ising model and the FFM, but it decreases with the system size, going from $18 \%$ at $L=30$ to $7 \%$ at $L=400$.
[1] A. Johanses, O. Ledoit, and D. Sornette, Crashes as critical points, Int. J. Theor. Appl. Finan. 03, 219 (2000).

[2] A. E. Biondo, A. Pluchino, and A. Rapisarda, Modeling financial markets by self-organized criticality, Phys. Rev. E 92, 042814 (2015).

[3] V. Filimonov and D. Sornette, Apparent criticality and calibration issues in the Hawkes self-excited point process model: Application to high-frequency financial data, Quant. Finan. 15, 1293 (2015).

[4] D. Sornette, Why Stock Markets Crash-Critical Events in Complex Financial Systems (Princeton University Press, Princeton, NJ, 2017).

[5] O. Peters and J. D. Neelin, Critical phenomena in atmospheric precipitation, Nat. Phys. 2, 393 (2006).

[6] J. Yano, C. Liu, and M. W. Moncrieff, Self-organized criticality and homeostasis in atmospheric convective organization, J. Atmos. Sci. 69, 3449 (2012).

[7] A. M. Selvam, Self-organized Criticality and Predictability in Atmospheric Flows (Springer International Publishing, Cham, 2017).

[8] L. Cocchi, L. L. Gollo, A. Zalesky, and M. Breakspear, Criticality in the brain: A synthesis of neurobiology, models and cognition, Progr. Neurobiol. 158, 132 (2017).

[9] J. Hesse and T. Gross, Self-organized criticality as a fundamental property of neural systems, Front. Syst. Neurosci. 8, 166 (2014).

[10] K. K. Iyer, Sleep, wake, and critical brain states: Corollaries from brain dynamics, Front. Neurosci. 12, 948 (2018).

[11] L. Brochini, A. de Andrade Costa, M. Abadi, A. C. Roque, J. Stolfi, and O. Kinouchi, Phase transitions and self-organized criticality in networks of stochastic spiking neurons, Sci. Rep. 6, 35831 (2016).

[12] D. Sornette, Critical Phenomena in Natural Sciences (SpringerVerlag, Berlin, 2006).

[13] D. Markovic and C. Gros, Power laws and self-organized criticality in theory and nature, Phys. Rep. 536, 41 (2014).

[14] G. Pruessner, Self-Organised Criticality: Theory, Models and Characterisation (Cambridge University Press, Cambridge, 2012).
[15] K. Christensen and N. R. Moloney, Complexity and Criticality (Imperial College Press, London, 2005).

[16] A. C. Staver, G. P. Asner, I. Rodriguez-Iturbe, S. A. Levin, and I. P. J. Smit, Spatial patterning among savanna trees in highresolution, spatially extensive data, Proc. Natl. Acad. Sci. USA 116, 10681 (2019).

[17] C. L. Alados, Y. Pueyo, D. Navas, B. Cabezudo, A. Gonzalez, and D. C. Freeman, Fractal analysis of plant spatial patterns: A monitoring tool for vegetation transition shifts, Biodiv. Conserv. 14, 1453 (2005).

[18] R. T. Wicks, M. J. Owens, and T. S. Horbury, The variation of solar wind correlation lengths over three solar cycles, Sol. Phys. 262, 191 (2010).

[19] P. Expert, R. Lambiotte, D. R. Chialvo, K. Christensen, H. J. Jensen, D. J. Sharp, and F. Turkheimer, Self-similar correlation function in brain resting-state functional magnetic resonance imaging, J. R. Soc. Interface 8, 472 (2011).

[20] L. Palmieri and H. J. Jensen, The emergence of weak criticality in SOC systems, Europhys. Lett. 123, 20002 (2018).

[21] V. Privman, P. C. Hohenberg, and A. Aharony, Phase Transitions and Critical Phenomena (Academic Press, San Diego, CA, 1991).

[22] K. Christensen, N. Farid, G. Pruessner, and M. Stapleton, On the scaling of probability density functions with apparent power-law exponents less than unity, Eur. Phys. J. B 62, 331 (2008).

[23] E. Ising, Beitrag zur theorie des ferromagnetismus, Z. Phys. 31, 253 (1925).

[24] S. G. Brush, History of the Lenz-Ising model, Rev. Mod. Phys. 39, 883 (1967).

[25] L. Onsager, Crystal statistics. I. A two-dimensional model with an order-disorder transition, Phys. Rev. 65, 117 (1944).

[26] U. Wolff, Collective Monte Carlo Updating for Spin Systems, Phys. Rev. Lett. 62, 361 (1989).

[27] W. Heisenberg, Zur theorie des ferromagnetismus, Z. Phys. 49, 619 (1928).

[28] P. M. Chaikin and T. C. Lubensky, Principles of Condensed Matter Physics (Cambridge University Press, Cambridge, 1995). 
[29] J. M. Kosterlitz and D. J. Thouless, Ordering, metastability and phase transitions in two-dimensional systems, J. Phys. C: Solid State Phys. 6, 1181 (1973).

[30] P. Olsson, Monte Carlo analysis of the two-dimensional $X Y$ model. II. Comparison with the Kosterlitz renormalizationgroup equations, Phys. Rev. B 52, 4526 (1995).

[31] W. Janke and K. Nather, High-precision Monte Carlo study of the two-dimensional $X Y$ Villain model, Phys. Rev. B 48, 7419 (1993).

[32] M. Hasenbusch, The two-dimensional XY model at the transition temperature: A high-precision Monte Carlo study, J. Phys. A: Math. Gen. 38, 5869 (2005).

[33] J. M. Kosterlitz, The critical properties of the two-dimensional XY model, J. Phys. C: Solid State Phys. 7, 1046 (1974).

[34] B. Drossel and F. Schwabl, Self-Organized Critical Forest-Fire Model, Phys. Rev. Lett. 69, 1629 (1992).
[35] P. Grassberger, On a self-organized critical forest-fire model, J. Phys. A 26, 2081 (1993).

[36] S. Clar, B. Drossel, and F. Schwabl, Scaling laws and simulation results for the self-organized cirtical forest-fire model, Phys. Rev. E 50, 1009 (1994).

[37] K. Schenk, B. Drossel, S. Clar, and F. Schwabl, Finite-size effects in the self-organized critical forest-fire model, Eur. Phys. J. B 15, 177 (2000).

[38] G. Pruessner and H. J. Jensen, Broken scaling in the forest-fire model, Phys. Rev. E 65, 056707 (2002).

[39] P. Grassberger, Critical behavior of the Drossel-Schwabl forest fire model, New J. Phys. 4, 17 (2002).

[40] C. L. Henley, Statics of a "Self-Organized" Percolation Model, Phys. Rev. Lett. 71, 2741 (1993).

[41] A. Honecker and I. Peschel, Length scales and power laws in the two-dimensional forest-fire model, Physica A 239, 509 (1997). 